\begin{tabular}{c|c|c}
\hline \hline $\begin{array}{c}\text { Vol. 35(3):239-247 } \\
\text { http://dx.doi.org/10.4217/OPR.2013.35.3.239 }\end{array}$ & Ocean and Polar Research & September 2013 \\
\hline
\end{tabular}

Note

\title{
Automatic Estimation of Artemia Hatching Rate Using an Object Discrimination Method
}

\author{
Sung $\mathrm{Kim}^{1}$ and Hong-Yeon $\mathrm{Cho}^{2 *}$ \\ ${ }^{1}$ Marine Ecosystem Research Division, KIOST \\ ${ }^{2}$ Marine Environments \& Conservation Research Division, KIOST \\ Ansan 426-744, Korea
}

\begin{abstract}
Digital image processing is a process to analyze a large volume of information on digital images. In this study, Artemia hatching rate was measured by automatically classifying and counting cysts and larvae based on color imaging data from cyst hatching experiments using an image processing technique. The Artemia hatching rate estimation consists of a series of processes; a step to convert the scanned image data to a binary image data, a process to detect objects and to extract their shape information in the converted image data, an analysis step to choose an optimal discriminant function, and a step to recognize and classify the objects using the function. The function to classify Artemia cysts and larvae is optimally estimated based on the classification performance using the areas and the plan-form factors of the detected objects. The hatching rate using the image data obtained under the different experimental conditions was estimated in the range of $34-48 \%$. It was shown that the maximum difference is about $19.7 \%$ and the average root-mean squared difference is about $10.9 \%$ as the difference between the results using an automatic counting (this study) and a manual counting were compared. This technique can be applied to biological specimen analysis using similar imaging information.
\end{abstract}

Key words : Artemia, cyst and larvae, hatching rate, image analysis, discriminant analysis

\section{Introduction}

Brine shrimp (Artemia sp.) belongs to the phylum Arthropoda, class Crustacea. These organisms are widely used as live food for larvae in crustacean and marine finfish farming (Treece 2000). Furthermore, Artemia have been utilized as an in vivo model species for biological responses (mainly for the hatching rate) corresponding to increased carbon dioxide concentrations in the ocean as well as biological toxicological experiments as it only takes $24 \mathrm{hr}$ for larvae to hatch from cysts (Brix et al. 2006; Onocha et al. 2011; Salma et al. 2012). Hatching rate is usually calculated by manual counting aliquots from a hatching tank (Salma et al. 2012). However, the number of observed samples must be large and experiments should be repeated many times to decrease the margin of

\footnotetext{
*Corresponding author. E-mail : hycho@kiost.ac
}

error of such biological experiments. But, the manual counting of additional specimens requires increased time and cost (Bates and Tiersch 1997). Therefore, automatic hatching rate estimations using recently developed imaging analysis techniques are needed to decrease costs and increase accuracy.

Digital imaging processing (DIP) using photo and video image data have been utilized in various marine fields to measure the distribution of chlorophyll-a concentration and shoreline changes, as well as wave height (Kim and Cho 2005; Kim et al. 2008).

These digital imaging analysis techniques have also been used to identify phytoplankton (Tsuji and Nishikawa 1984; Brown et al. 1989), to count cysts and larvae numbers (Been et al. 1996; Friedland et al. 2005), to analyze the morphological information of marine organisms (length and area) (Cardin and Friedland 1999; Thorsen and Kjesbu 2001; Brillon et al. 2005; Rideout et al. 2005), and 
to measure the shape parameters of fish's otoliths.

The major field of the DIP application is the automatic counting of the cyst, larva, and plankton numbers that have relatively simple classification criteria and require repetition. However, image analysis basically recognize the objects and only provide shape information for the recognized objects (or color information); preliminary information is needed to develop criteria extraction processes for classifying different objects or connecting objects to information from recognized objects.

Therefore, criteria for classifying cysts and larvae from recognized objects in Artemia imaging data are proposed in the present study.

As a result, Artemia cysts and larvae were classified and counted faster and easier to measure hatching rates.

\section{Materials and methods}

\section{Materials}

Hatching rate was measured utilizing an image processing technique that automatically classified the cysts and larvae and counted them on color imaging data from an Artemia cyst hatching experiment. The imaging data were obtained using the following procedures. Artemia cysts were added to $1.8 \mathrm{~L}$ bio-assay tanks (seawater salinity $30.0 \mathrm{PSU}$; air injection volume $1,000 \mathrm{~cm}^{3} / \mathrm{min}$ ) rectangular plastic water bath and incubated at room temperature for 46 hours. The amount of Artemia cysts was $0.121-0.137 \mathrm{~g}$ (mean, 0.129 $\pm 0.005 \mathrm{~g})$. The temperature of the hatching water bath was maintained at $26.5-26.9^{\circ} \mathrm{C}$ (mean, 26.7 $7^{\circ} \mathrm{C}$ ). The unhatched cyst and hatched larvae imaging data were

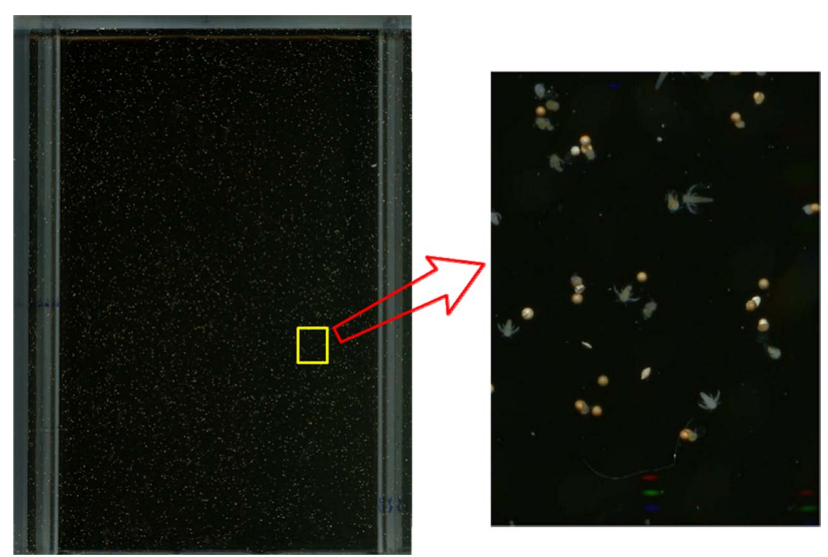

Fig. 1. Original color image data (full and zoomed partial images). The size of the zoomed partial image covers about $0.1 \%$ of the full image. Mixture of objects including cysts, larvae, and other meaningless objects collected using a renovated scanner after cyst shells floating on the surface were removed. A scanner (Epson Perfection V700 PHOTO model) was used with a resolution of 2,400 dpi. Because the rectangular box area was scanned, the pixel size of the scanned image area was $20,400 \times 28,079$, and the actual size of a pixel was approximately $0.01 \mathrm{~mm}$ (Fig. 1).

As indicated in Fig. 1, the scanned color image data of Artemia includes various kinds of significant objects, such as unhatched cysts (eggs), hatching cysts, larvae in the early and late stages of hatching larvae, and various cystslarvae touching each other. There are also many meaningless objects, such as small point objects, slightly-long line objects, and different kinds of unidentifiable objects having indistinct boundaries.

\section{Methods}

Three steps were used to estimate the Artemia hatching rate. In the first step, the scanned original image was converted to a gray scale image. The gray scale image was converted to a black and white binary image to separate the objects from the background gray scale image. Then, the image was converted into a final binary image to remove meaningless small objects and create boundaries using a morphological calculation technique. Image processing was carried out using the MATLAB Digital Image Processing Toolbox (MathWorks 2012) and a basic image processing technique (Sonka et al. 1999; Gonzalez and Wintz 1987) that included various image analysis functions. The detailed procedures were as follows.

(1) Convert the color image to a gray scale image (In cases the separation was performed based on object recognition and shape but not color, a black and white image was analyzed during the separation procedure).

(2) Convert the image data to binary image data by dividing the background and the objects (black and white binary image) using the widely-used Otsu method.

(3) Obtain a final image by conducting boundary image processing to remove the boundary and object noise.

The second step was a specimen analysis procedure to extract a partial image (a type of specimen image) from the entire image at random. A partial image can be regarded as a specimen (sample) and its size is about $0.1 \%$ of the full image. First, the morphological parameters of the detected objects were obtained in the final binary image data converted from the sample image. The objects of the partial image are also manually classified as the various cyst and larval groups. Based upon the automatically 
extracted parameters and manually classified grouping information of the objects, the optimal discriminant functions were determined for the partial image data set. This functions are used to separate the objects as the unhatched cysts and the larvae in the early hatching stage (cyst shell was attached), the young (partially hatched) and completely hatched larval stage (cyst shell was not present), and the others. The other objects include the cysts and larvae touching each other and meaningless objects not removed in the first step. The objects of the cysts and larva touching each other were classified as the 3 different groups (touching by cysts only, both cysts and larvae, and larvae only). All detected objects were classified by a predetermined linear discriminant equation and then the difference caused by the object discrimination was analyzed.

In the third step, the black and white binary image conversion drawn in the first step and the classification criteria in the second step were applied to an entire image. In this stage, the entire image can be regarded as the population. Every detected object in the image were classified based upon the discriminant function and a hatching rate was computed by counting the object numbers of the subdivided groups of the unhatched cysts, hatching cysts, and larvae in the early and late hatching stages. The hatching rate was calculated as follows.

Where,

$N=$ number of recognized (detected) objects in total image area

$N u=$ number of objects that were not relevant to Artemia hatching or that were unidentified among the recognized objects

$N o=$ number of objects touching each other among the recognized objects

No_egg = number of cysts among the objects touching each other (computed from the classification of the $\mathrm{No}$ objects)

No larvae $=$ number of larvae among the objects touching each other (computed from the classification of the No objects; No is approximately half of the No_egg + No_larvae)

$N \_$egg $=$number of cysts among the recognized individual objects

$N$ larvae $=$ number of larvae among the recognized individual objects

Therefore, the following equation was established:

$N-N u-N o=N \_$egg $+N \_$larvae (number of eggs and larvae excluding eggs and larvae touching each other)
$T N \_$egg $=$total number of recognized eggs among the individual and touching objects $=N \_$egg $+N o \_e g g$

$T N$ larvae $=$ total number of recognized larvae among the individual and touching objects $=N$ larvae + No_larvae

Hatching rate $\left(R_{H}\right)$ was calculated with the following equation:

$$
R_{H}=T N \_ \text {larave } /\left(T N \_ \text {egg }+T N \_ \text {larvae }\right)
$$

In general, the hatching cysts were counted as the $N$ larvae even though they could be classified as partially hatched larvae.

The hatching rate was calculated using the detailed cyst and larvae classification information. Limitations and errors while establishing the criteria to classify the cyst and larvae hatching steps occurred due to the continuous nature of hatching but were omitted from the present study.

\section{Results and discussion}

\section{Conversion of image data (first step)}

The results of the first step of the image analysis were related to the computational efficiency of the analysis. The effects of each step in detail were examined using a sample partial image. In general, an analysis was performed by converting the color image to a gray scale image except when color information was necessary to make a judgment (Rhee 2004). As shape information was more important than color information in the present study, the analysis was carried out by converting all images to gray scale. The Artemia image during hatching was obtained by removing small unconfirmed meaningless objects and conducting a boundary process utilizing a grayscale image, a black and white binary image, and a morphological calculation (Fig. 2). The object was recognized more clearly in the converted image and the gray scale image (Fig. 2a). Meaningless noises as well as meaningful objects were present while converting the images to black and white binary images (Fig. 2b) which determined the object boundary by separating the background from an object. The small gaps and boundaries of the noise and object were removed by performing morphological operations (an opening operation was carried out in the present study). Most small meaningless objects were successfully removed in the final black and white binary image (Fig. 2c) after carrying out the calculation. Larger or long impurities were removed by applying more structural 


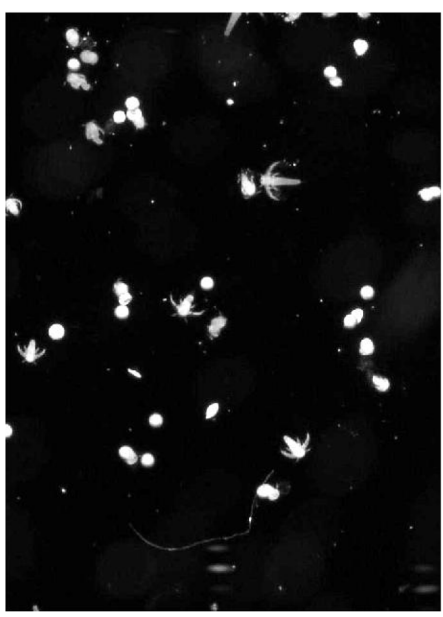

(a)

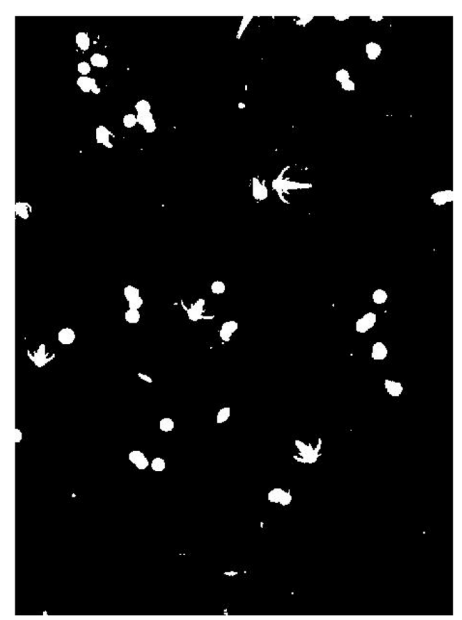

(b)

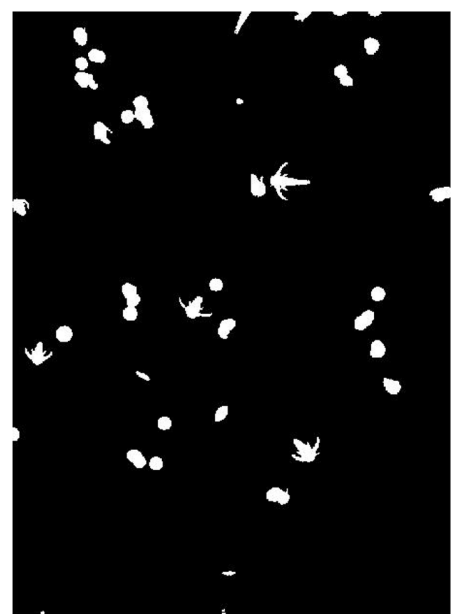

(c)

Fig. 2. An image converted from a scanned image; (a) Gray scale image data (partial), (b) Black and white binary image (partial), and (c) An image treated to remove unidentified objects and the boundary using the morphological calculation function

elements during an erosion and a dilation operations; however, only objects with $<50$ pixels $(=0.5 \mathrm{~mm})$ in radius were selected and removed. Although these calculations were effective to smooth the boundary or process a small gap, an excess applying of the morphological operations was inappropriate because these calculations may change actual image boundaries (Gonzalez and Wintz 1987).

\section{Object recognition and shape information extraction (second step)}

Shape metrics defined as the various length combinations are widely used to classify objects based on shape (Jiao et al. 2012; Zorica et al. 2010; Imasogie and Wendt 2004; $\mathrm{Hu}$ and Stroeven 2006; Arasan et al. 2010). The shape metrics (information) of each object were extracted using the finally converted black and white binary image obtained from the first step. A serial number was given to the each recognized object, and the values of the circular degree $\left(\mathrm{PF}\right.$, plan-form factor $\left.=4 \pi \mathrm{A} / \mathrm{P}^{2}\right)$, defined as the function of perimeter $(\mathrm{P})$ and area (A, pixel numbers) in the object among the shape information, were displayed as shown in Fig. 3. The form factor was close to 1.0 and 0.2-0.3 in cases where the objects were round (circle) such as an Artemia cyst and a complex shape such as larvae, respectively. Two types of object shape information were extracted: one is the absolute size information including the perimeter, area, and length of the major axis and the minor axis and the other is the relative ratio information including the plan-form factor, solidity, and

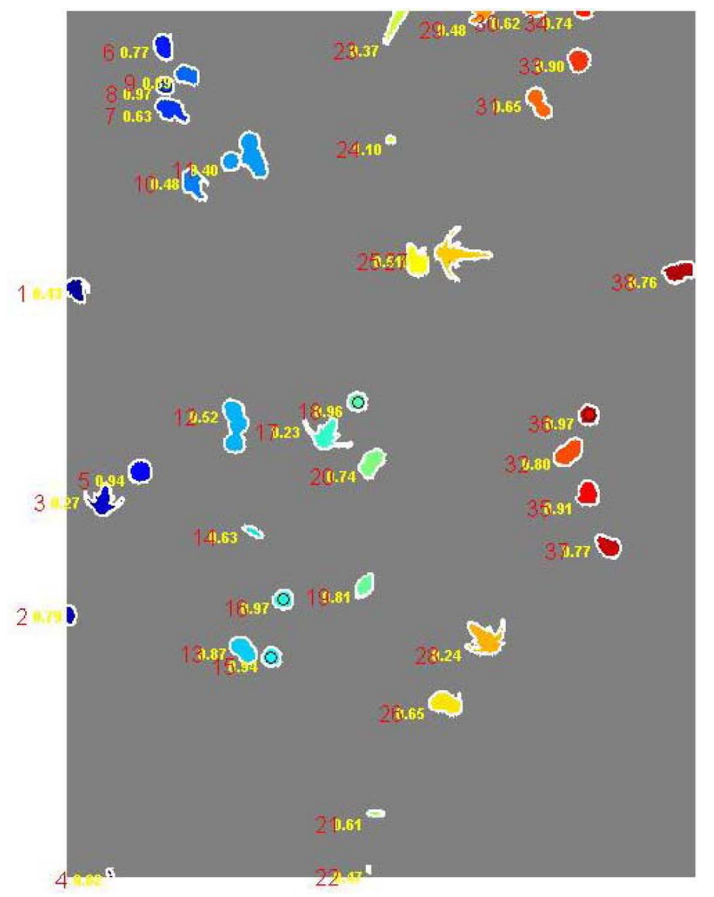

Fig. 3. Shape metrics and boundary tracks of the detected objects (area, length, etc.)

eccentricity by combining the absolute information. The shape information of each object can be used as a criterion to separate an object, and the shape information is determined based upon 1 pixel $=1$ unit. The combined criterion of absolute and relative information was utilized in this study. This object classification method using a 


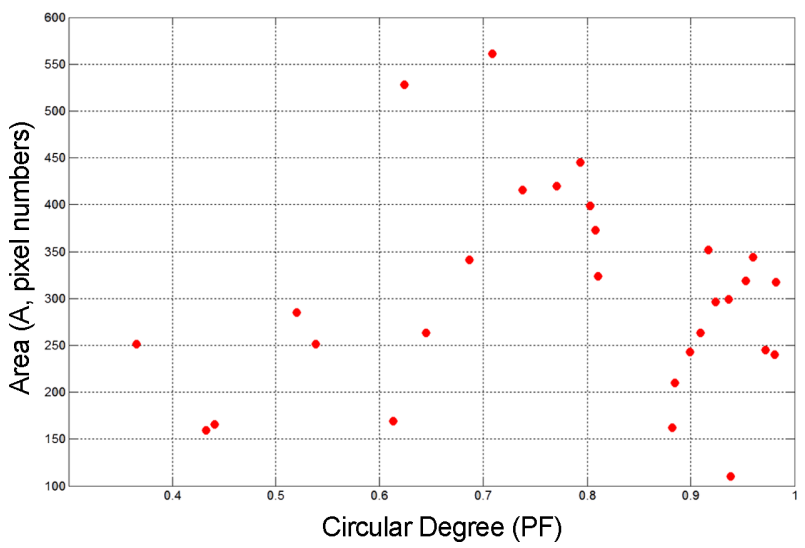

Fig. 4. Scatter plot of the area and form factor of the detected objects

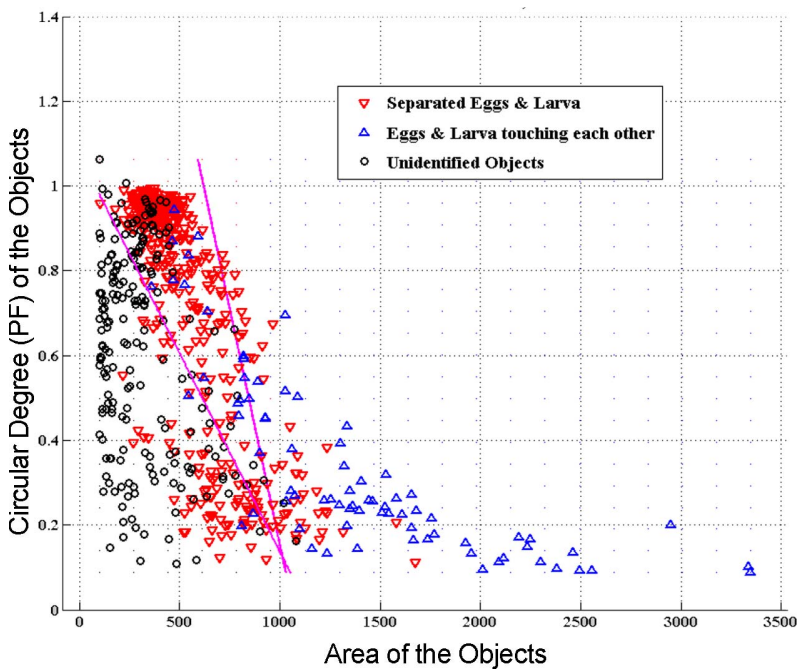

Fig. 5. Separate functions for Artemia cysts and larvae, overlapped objects, and unidentified objects based on the partial training set image

form factor or solidity and area or length information as a separation criterion is widely used in shape classification and characterization studies ( $\mathrm{Hu}$ and Stroeven 2006; Zorica 2010). In the scatter plot between an area and circular degree of the objects (Fig. 4), Artemia cysts were positioned around 1.0 circular degree and larvae were positioned below about 0.5 circular degrees.

Criteria rules or functions for classifying cysts and larvae using shape information are better if they are simple and the performance acceptable. The objects were separated into seven categories in this study. First, cysts and larvae were selected by extracting and applying the criteria to classify and remove the objects touching each other and meaningless small objects. For the first time, the cysts and larvae can be classified using a circular degree

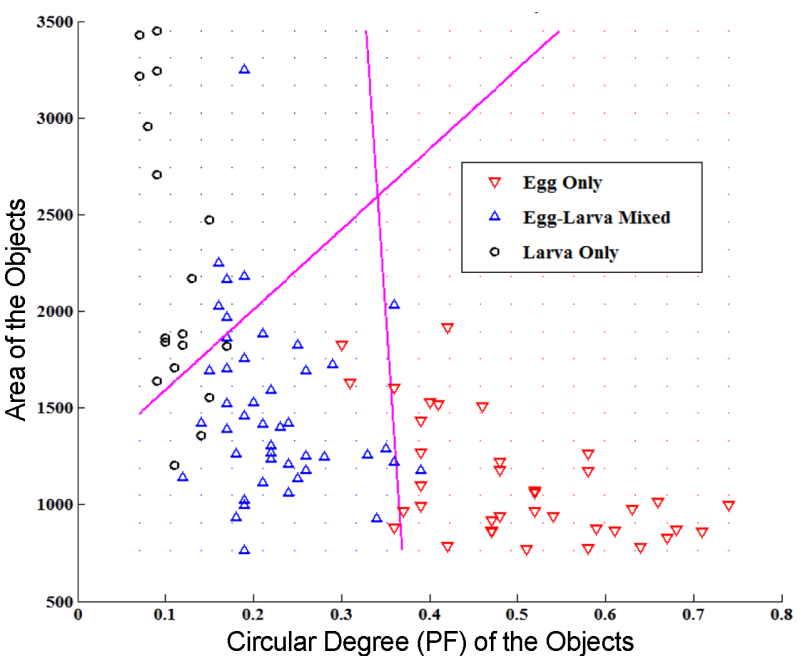

Fig. 6. Overlap type classification function for overlapped objects based on the partial training set image

only. Although area could also be used, the cysts and larvae were classified very accurately (approximately 95\% accuracy) using the 0.5 form factor criterion (Fig. 5). Cysts and cysts during the early stage of hatching could also be separated by applying the $0.85-0.90$ section form of the factor criterion even if separation accuracy is significantly decreased.

Fig. 6 indicates that Artemia objects touching each other and meaningless unconfirmed objects can be separated using the linear function by applying the area and form factor. A classification method is available to use the optimum function drawn from the assumptions of a normal distribution or a specified distribution function (Sonka et al. 1999); however, a simple linear function for the separation can be created using the classification symbol based on separate shape information if the object classification is simple. In this study, the linear function of the simple separation criterion was drawn as shown below. Finally, the drawn object classification function and classification error are as follows.

\section{A function to classify the Artemia cysts and larvae objects touching each other, the other large objects, and the unidentified small meaningless objects}

The unidentified objects (very small and extremely long objects, impurities, and deformed cyst shells, etc.) and overlapping objects were classified using an optimum linear function. The classification function is indicated below, and the classification error was $27 \%$ (mean $\log \mathrm{p}=$ -18.2). 


$$
\text { Eq. (1) } \begin{aligned}
\mathrm{f}_{1}(\mathrm{PF}, \mathrm{A})= & -11.5100+1.5071 \times \mathrm{PF}+11.1946 \\
& \times 10^{-3} \times \mathrm{A} \\
\text { Eq. (2) } \mathrm{f}_{2}(\mathrm{PF}, \mathrm{A})= & -6.5432+6.0793 \times \mathrm{PF}+5.7020 \\
& \times 10^{-3} \times \mathrm{A}
\end{aligned}
$$

Here, PF and A indicate the form factor and the area of the recognized objects, respectively.

Therefore, if the objects satisfy $f_{1}>0$ condition, the Artemia cysts and larvae are classified as the objects touching each other, whereas if they satisfy $\mathrm{f}_{2}<0$ condition, they are classified as unconfirmed objects (meaningless objects, impurities, and deformed cyst shells). The remaining objects were separated as Artemia cysts and larvae. However, in the case of the $\mathrm{PF}<0.6$ condition, the specified object area, 500, was used as the criteria area ( $<500$ was considered impurities and meaningless objects) to decrease the total classification error in the case of $\mathrm{f}_{2}<0$.

\section{Classification functions for the objects touching each other}

Cyst and larvae were often attached to the objects touching each other and these objects were roughly classified into three types as follows. The classification function is indicated below, and the classification error was approximately $15 \%$ (mean $\log p=-10.7$ ).

$$
\begin{aligned}
& \text { Eq. (1) } \mathrm{f}_{1}(\mathrm{PF}, \mathrm{A})=-15.5766+40.8785 \times \mathrm{PF}+0.6234 \\
& \times 10^{-3} \times \mathrm{A} \\
& \text { Eq. (2) } \mathrm{f}_{2}(\mathrm{PF}, \mathrm{A})=-3.1598-11.1258 \times \mathrm{PF}+2.6808 \\
& \times 10^{-3} \times \mathrm{A}
\end{aligned}
$$

Therefore, if the object satisfies $f_{1}>0$ condition, the object can be regarded as the object attached to two Artemia cysts only, and if it satisfies $\mathrm{f}_{2}>0$ condition, the object can be regarded as the object formed with two larva. The others are the objects attached to a cyst and larvae. If the areas of the objects are $>1,000,1,500$, or 2,000 (pixels), the objects are assumed as the objects formed with more than three cysts or larva. However, these objects rarely occurred, so we assumed every object is formed with only two meaningful objects (combination of the cyst and larva).

\section{Classification of Artemia cysts and larvae}

The objects different from the objects touching each other and unconfirmed objects (meaningless objects) can be considered as the Artemia cysts and larvae. In this study, objects were specified in detail, such as unhatched cysts, the early hatching stage (E-1, E-2), and larval stage (INSTAR-1 and V) (Fig. 7); however, the separation error

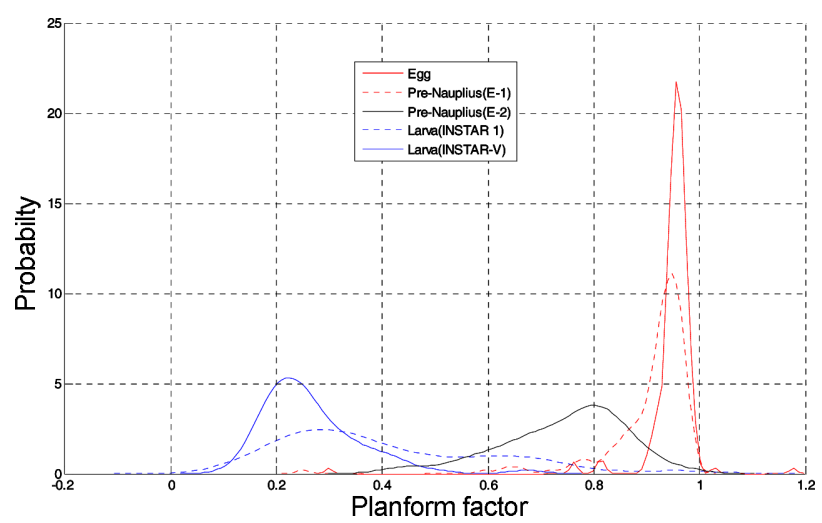

Fig. 7. A distribution function of occurrence frequency of Artemia cyst and larvae based on the circular degree only

for the cysts and the early hatching stage becomes large, whereas the separation error for the cysts and larvae was very low $(<10 \%)$. Thus, only the form factor was used as a separation criterion and the specific PF values, 0.9 and 0.5 , were applied for each case. In other words, the objects are classified as cysts in the case of the $\mathrm{PF}>0.9$, as the early-hatching stage larvae lying between the hatching stage and a larval stage in the case of the $0.5<\mathrm{PF}<0.9$, and the fully-hatched larvae in the case of the $\mathrm{PF}<0.5$ conditions, respectively. The separation error was not negligible; however, the final counting error decreases, as it reflects the actual level of the offset for error compensation. The numbers of the misclassified objects based on the criteria decreased in cases of counting the correct objects as the wrong ones, whereas they increased in cases of counting the wrong objects as the correct ones.

\section{Hatching rate calculation (third step)}

The hatching rate was calculated using the meaningful objects count information. The pre-determined separation function was applied to count the objects in the entire scanned images of an Artemia hatching stage created under different experimental conditions. Eight images were used for the hatching rate calculation from the different $p \mathrm{CO}_{2}$ condition in the bio-assay tank each other. As the objective of the present study was a performance evaluation of the hatching rate. The hatching rates for every image data were estimated under the assumption that each image is the independent image of the different conditions. Table 1 represents the number of different objects used in the hatching rate estimation and the final calculated hatching rate. The counting error based upon 
Table 1. Hatching rate estimate and object counting detected from the Artemia image data

\begin{tabular}{|c|c|c|c|c|c|c|c|c|}
\hline Image No. of Bioassay & 1 & 2 & 3 & 4 & 5 & 6 & 7 & 8 \\
\hline $\bar{N}$ & 35,298 & 41,913 & 44,521 & 44,571 & 39,691 & 52,966 & 38,311 & 50,527 \\
\hline$N u$ & 4,618 & 6,740 & 10,648 & 9,682 & 5,904 & 14,088 & 6,382 & 15,768 \\
\hline No & 1,788 & 2,982 & 3,652 & 3,979 & 6,523 & 4,039 & 3,302 & 6,652 \\
\hline$N \_$egg $+N \_l a r v a e(=N-N u-N o)$ & 28,892 & 32,191 & 30,221 & 30,910 & 27,264 & 34,839 & 28,627 & 28,107 \\
\hline$T N \_$egg (automatic) & 17,054 & 22,673 & 22,555 & 21,313 & 26,482 & 26,552 & 21,939 & 25,957 \\
\hline$N$ plarvae & 10,320 & 7,109 & 4,405 & 4,263 & 2,661 & 9,673 & 6,884 & 2,450 \\
\hline$T N \_l a r v a e^{*}$ & 5,095 & 8,373 & 10,564 & 13,293 & 11,168 & 6,693 & 6,407 & 13,004 \\
\hline TN_larvae (automatic) & 15,415 & 15,482 & 14,969 & 17,556 & 13,829 & 16,366 & 13,291 & 15,454 \\
\hline$T N \_e g g+T N \_l a r v a e$ & 32,469 & 38,155 & 37,524 & 38,869 & 40,311 & 42,918 & 35,230 & 41,411 \\
\hline$H_{R}(\%)$ (automatic) & 47.5 & 40.6 & 39.9 & 45.2 & 34.3 & 38.1 & 37.7 & 37.3 \\
\hline$T N \_$egg (manual) & 23,586 & 25,885 & 23,609 & 21,738 & 22,688 & 28,442 & 27,891 & 19,528 \\
\hline TN_larvae (manual) & 18,871 & 20,320 & 17,958 & 14,154 & 14,193 & 17,792 & 16,248 & 13,456 \\
\hline$H_{R}(\%)$ (manual) & 27.8 & 27.6 & 36.8 & 42.4 & 40.1 & 31.1 & 27.1 & 50.6 \\
\hline
\end{tabular}

Ref. All variables in the Table are defined in Section 2. automatic = count and estimation using the method suggested in this study, manual = count and estimation manually, $T N_{-}$larvae ${ }^{*}=$ no. of larvae (does not include the partially hatched cysts); $N$ plarvae $=$ no. of the partially hatched larvae. $T N \_l a r v a e=T N \_$larvae ${ }^{*}+\bar{N} \_$plarvae $)$

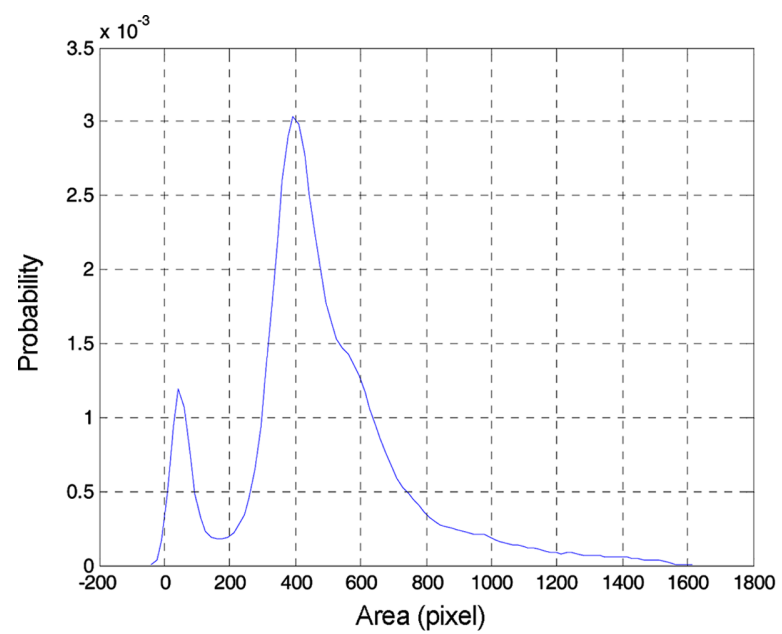

(a)

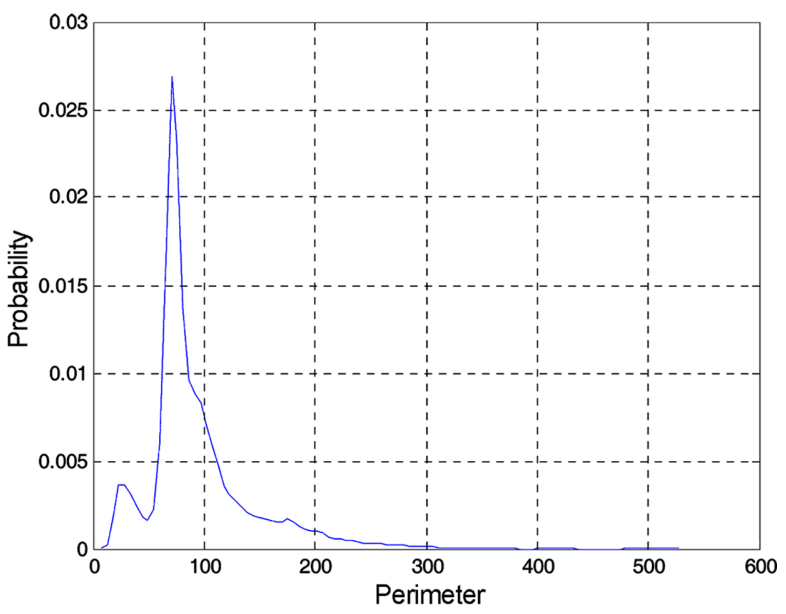

(b)

Fig. 8. Distribution of the shape metrics of Artemia cysts, larvae, and other objects; (a) Area probability distribution of all objects detected in image no. $1(n=19,767)$ and $(b)$ Length probability distribution of all objects detected in image no. $1(n=19,767)$

the object classification was considered as the error calculated from the object classification criterion in the second step.

The hatching rate would be calculated faster and more accurately in bioassay experiments under various experimental conditions if better resolved image data and shape information are utilized to enable object classification with better accuracy. The hatching rate was $34-48 \%$ when estimated using the automated counting technique developed in this study. The maximum error and average root mean squared error (or difference in some sense) were 19.7\% and $10.9 \%$, respectively, when compared the Artemia larvae count results estimated manual counting with automatic counting using the classification criteria function.

The reasons for such errors might be due to the spatial variations (non-homogeneous scattering) for the manual counting method and the grouping errors for the cysts and early hatching stage cysts during the automatic counting. The manual counting method covers just about the $7-10 \%$ of the entire image areas. The manual count results were adjusted to the results for the entire area. The automatic counting results were also adjusted to the value for an 
entire area from the pre-specified area results excluding the adjacent part around the boundary of the entire image to decrease boundary error.

Because the image analysis results provide hatching rate information as well as shape information of each object, a statistical distribution and object shapes for each type can be obtained (e.g., the area distribution and perimeter of multiple Artemia cysts and larvae, Fig. 8). Although the pixel numbers should be high to express the size of an object accurately, if the actual size of an object is small, expressing pixel number would be too small under fixed resolution conditions; thus, imaging analysis should be conducted using image information with appropriate resolution that provides shape information including length and area. Image pixel numbers for objects analyzed in the study were $>300$ and 500 for the cysts and larvae, respectively, which did not deviate from the object shape discrimination criteria (Sonka et al. 1999).

\section{Conclusion and suggestion}

We developed an efficient method to count cysts and larvae automatically using the object discrimination function from the Artemia image data.

Based on this technique, the hatching rate of the species was estimated using Artemia image data from various experimental conditions. The hatching rate was $34-48 \%$ when estimated using the automated counting technique developed in this study. The maximum error and average root mean squared error were $19.7 \%$ and $10.9 \%$, respectively, when compared manual counting with the automatic counting using the classification criteria function. The average difference of the hatching rate estimate was approximately $10 \%(\mathrm{p}=0.05)$ and was due to the error in the criteria for classifying Artemia cysts and larvae.

The discriminant function to classify Artemia cysts and larvae was combined with form factor $(=0.5)$, length, and area information. The best classification functions for the objects depend on the object shape characteristics, the area information for small objects, and the form factor information for the objects touching each other.

However, due to the limits of image resolution, relatively high errors were observed in clearly separating objects during the early cyst hatching phase because of the uncertain touching object area information. Although there were large deviations while separating details, we were able to clearly separate cysts and larvae with $>95 \%$ accuracy $(\mathrm{p}=0.05)$ because cysts and larvae are different shapes.
These techniques can be utilized for counting simple fields, extracting shape information, and for bioassay data analysis to classify objects.

Further investigations are warranted regarding the database for characteristic information and drawing the function for the shape and classification criteria.

\section{Acknowledgements}

We are grateful to the anonymous reviewers for their valuable comments and advice.

This work was supported by Ecological Impacts of Ocean Acidification (EcoAcid) program (PE98928) of Korea Institute of Ocean Science and Technology.

\section{References}

Arasan S, Hasiloglu AS, Akbulut S (2010) Shape properties of natural and crushed aggregate using image analysis. Int J Eng Res Appl 1(2):221-227

Bates MC, Tiersch TR (1997) Low-cost computer-assisted image analysis for fisheries research. Prog Fish Cult 59(3):235-240

Baxes GA (1984) Digital image processing: A Practical Primer. Cascade Press, New Jersey, $182 \mathrm{p}$

Been TH, Meijer EMJ, Beniers AE, Knol JW (1996) Using image analysis for counting larvae of potato cyst nematodes (Globodera spp.). Fund Appl Nematol 19(3): 297-304

Brillon S, Lambert Y, Dodson J (2005) Egg survival, embryonic development, and larvae characteristics of northern shrimp (Pandalus borealis) females object to different temperature and feeding conditions. Mar Biol 147:895-911

Brix KV, Gerdes RM, Adams WJ, Grosell M (2006) Effects of copper, cadmium, and zinc on the hatching success of brine shrimp (Artermia franciscana). Arch Environ Contam Toxicol 51:580-583

Brown LM, Gargantini I, Brown DJ, Atkinson HJ, Govindarajan J, Vanlerberghe GC (1989) Compter-based image analysis for the automated counting and morphological description of microalgae in culture. J Appl Phycol 1:211-225

Cadrin SX, Friedland KD (1999) The utility of image processing techniques for morphometric analysis and stock identification. Fish Res 43:129-139

Friedland KD, Ama-Abasi D, Manning M, Clarke L, Kligys G, Chambers RC (2005) Automated egg counting and sizing from scanned images: Rapid sample processing 
and large data volumes for fecundity estimates. J Sea Res 54:307-316

Gonzalez RC, Wintz P (1987) Digital image processing. Second Edition. Addison-Wesley Pub. Co., Tokyo, 503 p

$\mathrm{Hu}$ J, Stroeven P (2006) Shape characterization of concrete aggregate. Acta Stereol 25:43-53

Imasogie BI, Wendt U (2004) Characterization of graphite particle shape in spheroidal graphite iron using a computer-based image analyzer. J Mineral Mater Charact Eng 3(1):1-12

Jiao L, Liu Y, Li H (2012) Characterizing land-use classes in remote sensing imagery by shape metrics. ISPRS J Photogramm 72:46-55

Kim BO, Cho HY (2005) Image processing for video images of buoy motion. Ocean Sci J 40(4):213-220

Kim BO, Cho HY, Lim DI, Yoon GS, Oh IS, Park YA (2008) Nearshore wave measurement using single-video images of buoy motions. J Coastal Res 24(6):1481-1486

Letcher BH, Bengston A (1993) Effects of food density on growth and on patterns of prey depletion by larvae silverside fish, Menidia beryllina (Cope); a laboratory investigation with image analysis. J Exp Mar Biol Ecol 167:197-213

MathWorks (2012) Image processing toolbox ${ }^{\mathrm{TM}}$ User's Guide. The MathWorks, Inc. Natick, Massachusetts, 596 p

Onocha PA, Oloyede GK, Olasunkanmi GS (2011) Chemical composition, brine shrimp toxicity and free-radical scavenging activity of leaf essential oil of Acalypha Ornata (Hochst). Adv Environ Biol 5:188-193

Rhee PK (2005) Image processing and biometric recognition (In Korean). Hongneung Science Pub., Seoul, 256 p

Rideout RM, Trippel EA, Litvak MK (2005) Effects of egg size, food supply and spawning time on early life history success of haddock Melanogrammus aeglefinus. Mar Ecol-Prog Ser 285:169-180

Salma U, Uddowla MH, Lee G, Yeo Y, Kim HW (2012) Effects of $\mathrm{pH}$ change by $\mathrm{CO}_{2}$ induction and salinity on the hatching rate of Artemia franciscana. Fish Aquat Sci 15(2):177-181

Sonka M, Hlavac V, Boyle R (1999) Image processing, analysis, and machine vision. Second Edition. PWS Publishing, Pacific Grove, $770 \mathrm{p}$

Storbeck F, Daan B (2001) Fish species recognition using computer vision and a neural network. Fish Res 51:1115

Thorsen A, Kjesbu OS (2001) A rapid method for estimation of oocyte size and potential fecundity in Atlantic cod using a computer-aided particle analysis system. J Sea Res 46:295-308

Treece GD (2000) Artemia production for marine larvae fish culture. Southern Regional Aquaculture Center, SPAC Publication No. 702, 4 p

Tsuji T, Nishikawa T (1984) Automated identification of red tide phytoplankton Prorocentrum triestinum in coastal areas by image analysis. J Ocean Soc Jap 40:425-431

Wu J, Jones KB, Li H, Loucks OL (2006) Scaling and uncertainty analysis in ecology: methods and applications. Springer, $352 \mathrm{p}$

Zorica B, Sinovcic G, Cikes Kec V (2010) Preliminary data on the study of otolith morphology of five pelagic fish species from the Adriatic Sea (Croatia). Acta Adriat 51(1):89-96

Received Aug. 6, 2013

Revised Sep. 6, 2013

Accepted Sep. 11, 2013 